\title{
Some Aspects of the Nutrition of Lactobacillus fermenti 36 in the Tube Assay of Thiamine
}

\author{
Z. G. B A N H I D I
}

The Wenner-Gren Institute for Experimental Biology, University of Stockholm, Sweden

\begin{abstract}
Reinvestigating the nutritional requirement of $L$. fermenti 36 on different media, it was found that:

$a$. the alkali treated peptone is a dispensable supplement if the basal medium is completed with tryptophane, since tryptophane was shown to be an essential nutrient for $L$. fermenti.

b. by using weak inoculum, it was found that whole yeast extract and panthetine are both stimulatory for growth of $L$. fermenti.

c. charcoal treated yeast extract appeared to be inhibitory under similar conditions.

$d$. the inhibition with the latter and the stimulation with pantethine can be compensated by reducing the modium with cysteine and/or ascorbic acid.

e. with tryptophane and increased level of casein hydrolysate as sources for amino acids, as well as with cysteine and ascorbic acid, a semisynthetic medium can be prepared, which allows good growth and reliable uniform response toward thiamine.

$f$. the size of the inoculum is a crucial factor in the $L$. fermenti test and should be carefully adjusted turbidimetrically for each run.
\end{abstract}

The assay of thiamine seems to be still in its developing stage, and it is
difficult to find a reliable technique when designing a program of investiga-
tion of the occurrence of thiamine and its derivatives in extracts of complex
biological materials. Chemical methods can hardly be considered for such a
task except for analysis of relatively pure solutions of this vitamin. While
many different microbiological methods have been described for the analysis
of the total vitamin $\mathrm{B}_{1}$ content of food stuffs and other natural products,
Lactobacillus fermenti 36 was favored in most of the procedures ${ }^{1-8}$ and this
organism is still considered as a potential tool with the least failure when
thiamine is analyzed ${ }^{9}$. Nevertheless, owing to inconsistencies in the results,
several nutritional trials were performed in different laboratories in order to
minimize the anomalies in the behavior of Lactobacillus fermenti 36 in the thia-
mine test 3 , 7 , 10, 8 . In every work referred to, the original basal medium of
Sarett and Cheldelin 1 was accepted as a nutritional basis for $L$. fermenti.
This medium is already supplemented with a natural extract viz. alkali treated Acta Chem. Scand. 12 (1958) No. 3 
or photolysed peptone. This complex natural complement obscured the results in the usual nutritional experiments, which are rather informative if performed with pure synthetic (amino acid mixture) or semisynthetic (casein hydrolysate) media.

The present work deals with the establishment of some nutritional requirements of $L$. fermenti, the fulfillment of which renders this organism useful for the tube assay of thiamine in small amounts of tissue and organ extracts on a semisynthetic basal medium.

\section{METHODS}

Stock cultures of Lactobacillus fermenti 36 (ATCC 9833) were kept on Bacto Micro Assay Culture Agar, stored at $6-8^{\circ} \mathrm{C}$ after growth for $24 \mathrm{~h}$ at $35^{\circ} \mathrm{C}$. The stock cultures were "renewed" in every four to six weeks by transferring to a fortified litmus-skim milk medium 11*. Several agar subcultures were prepared from a milk tube after each "renewing", and used for the preparation of inocula or for stock cultures. The fortification of the agar medium with $10 \mu \mathrm{g}$ per liter thiamine ${ }^{3,5}$ was found to be unnecessary in our work, probably because of the more complete composition of the Micro Assay Culture Agar compared with the original yeast extract - dextrose agar, recommended in other methods $1,12,13$.

Inoculum was prepared by transfer from an agar subculture to Bacto Micro Inoculum Broth fortified with $10 \%$ tomato juice filtrate ${ }^{6}$. After $18 \mathrm{~h}$ at $35^{\circ} \mathrm{C}$ this was found to give abundant growth compared to unsupplemented broth (see Table 1) or that with other different supplements.

The time of incubation for the inoculum was originally set at $24 \mathrm{~h}$ by Sarett and Cheldelin ${ }^{1}$, but this period was decreased by others ${ }^{12,5}$. In this way the interference of bacteria already adapted to the breakdown products of thiamine could be avoided in the test, since the adaption was stated to occur only when cultures passed the limit of $18 \mathrm{~h}{ }^{1,5}$.

The cell suspension was washed twice in saline, as usual, and was used for inoculation within one hour. The density of inocula was adjusted turbidimetrically in every experiment and two or three uniformly administered drops of inoculum, respectively, were used for tubes with 7 or $10 \mathrm{ml}$ of final volume. Two main type of inocula were used in our experiments, the "weak inoculum" adjusted to $90 \%$ transmission and the "medium

Table 1. Growth of Lactobacillus fermenti 36 on Bacto Micro Inoculum Broth with and without addition of tomato juice filtrate. Turbidimetric readings after $18 \mathrm{~h}$ of incubation expressed in per cent of transmission $(\% \mathrm{~T})$ values representing means of triplicates.

\begin{tabular}{|l|c|c|c|}
\hline & Broth & Uncompleted & \multicolumn{2}{|c|}{ Reinforced with tomato juice } \\
\cline { 3 - 4 } & $1 \% \mathrm{v} / \mathrm{v}$ & $10 \% \mathrm{v} / \mathrm{v}$ \\
\hline $\begin{array}{c}\text { Readings against } \\
\text { blanks as 100 }\end{array}$ & 33 & 28 & 13.5 \\
\hline $\begin{array}{c}\text { Readings against in- } \\
\text { oculated uncomple- } \\
\text { ted broth as 100 }\end{array}$ & 100 & 79 & 37.5 \\
\hline
\end{tabular}

* Bacto Yeast Extract $5 \mathrm{~g}$; Bacto Liver $1.5 \mathrm{~g}$ (or Parke-Davis Liver Concentrate $0.5 \mathrm{ml}$ ); tomato juice filtrate (canned, unseasoned) $100 \mathrm{ml}$; glucose $2 \mathrm{~g}$; agar $1 \mathrm{~g}$; Tween $801 \mathrm{ml}$; litmus $1 \mathrm{~g}$ dissolved in $10 \mathrm{ml} 0.1 \mathrm{~N}$ sodium hydroxide and filtered; skim milk to $1000 \mathrm{ml} ; \mathrm{pH}$ should be adjusted carefully to 6.4 with $0.1 \mathrm{~N}$ hydrochloric acid. 
strength inoculum" with $40 \%$ T. Heavier inoculum was found to be unsatisfactory because of high blanks (cf. Sarett and Cheldelin ${ }^{1}$ ).

Techniques. Calibrated pyrex tubes of $18 \mathrm{~mm}$ diemeter were used for the test with 7 or $10 \mathrm{ml}$ final volume.

Media combined with standard or test solutions were autoclaved for $15 \mathrm{~min}$ at 1 atm. overpressure in small autoclaves, where the total time needed for heating and cooling does not exceed $45 \mathrm{~min}$. While only steaming was recommended by Sarett and Cheldelin ${ }^{1}$ in their original method, Fitzgerald and Hughes ${ }^{5}$ found no destruction of thiamine in assay tubes when autoclaved for 10 min at $10 \mathrm{lb}$. We found serious loss of thiamine activity if the vitamin was autoclaved in pure solutions or in buffer solutions at $\mathrm{pH}$ 6.4-6.6. However, rather good protection could be observed if thiamine was autoclaved together with the basal media, and a comparative test with steril-filtered standards showed no special advantage in the rutine test.

Incubation was made in a water bath at $35^{\circ} \mathrm{C}$ for $17-18 \mathrm{~h}$ except when noted otherwise.

Measurement of cell growth through turbidity was achieved by readings of per cent light transmission at $6000 \AA$ in a Coleman Universal Spectrophotometer.

Preparations. Bacto-Peptone and Bacto-Yeast Extract were used both in the untrea ted form as whole extracts or in form of preparations in which the thiamine content was minimized by chemical destruction or absorption, viz.:

alkali treated peptone ${ }^{14}$,

photolyzed peptone ${ }^{15,12}$,

Norit A (charcoal) treated yeast extract ${ }^{16}$,

sulphite treated yeast extract ${ }^{17}$,

which were prepared according to the original prescriptions quoted.

Basal media of several different compositions were used in this work, as noted below. The composition of a semisynthetic basal medium is given in Table 2. This medium represents the recommended final medium for the thiamine test and the results of our nutritional experiments were considered for its composition. Although incorporation of further supplements was found not to improve the turbidimetric method, in case of an acidimetric semimicro assay, a completed semisynthetic basal medium was used. According to the terminology used ir the description of our experiments, the medium presented herewith is a "high amino acid reduced semisynthetic medium".

In the course of the nutritional studies the following main types of media were substituted for the final basal medium, except when noted otherwise:

"Low amino acid medium" as in Table 2 with only 5 g per liter casein hydrolysate, without cysteine, ascorbic acid and pantethine.

"High amino acid medium" as above with 12 g casein hydrolysate.

"Reduced low amino acid medium" similar to the low amino acid medium but with $1 \mathrm{~g}$ of cysteine and $400 \mathrm{mg}$ of ascorbic acid.

"Reduced high amino acid medium" as above with $12 \mathrm{~g}$ casein hydrolysate.

Bacto Thiamine Assay Medium was used in the present experiments in place of the Sarett and Cheldelin medium despite small differences in the composition of the two media, such as the incorporation of photolysed peptone in the former, instead of alkali treated peptone as described for the original method. Actually the Bacto medium represents the standard medium used for present analytical methods ${ }^{12,5}$.

\section{EXPERIMENTS AND RESULTS}

$$
\text { Tryptophane requirement of Lactobacillus }
$$

In the original basal medium of Sarett and Cheldelin ${ }^{1}$ tryptophane is not incorporated, despite the fact that tryptophane is usually essential for Lactobacilli ${ }^{18}$ and is practically missing from acid hydrolyzed casein. It is to be mentioned that the same authors found tryptophane to be superfluous, similar

Acta Chem. Scand. 12 (1958) No. 3 
Table 2. Semisynthetic Basel Medium.

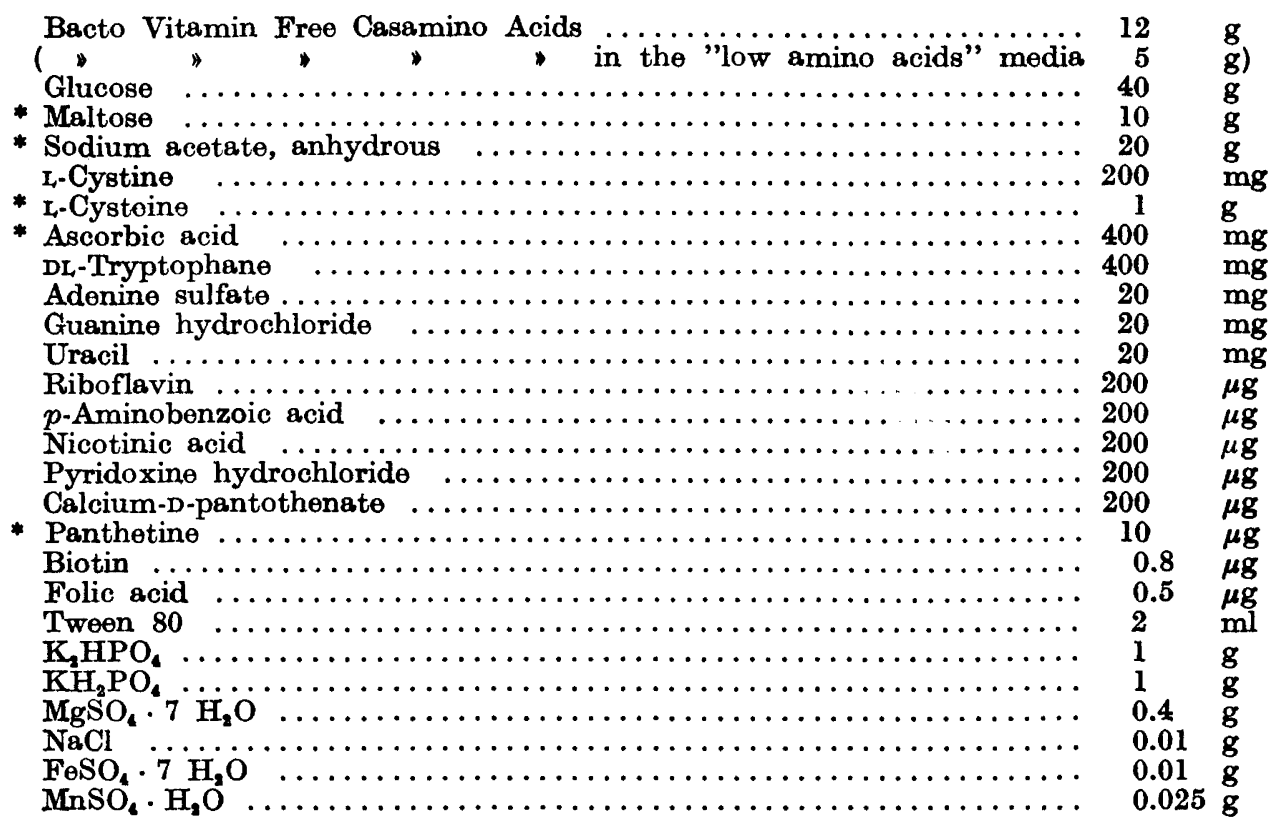

Distilled water to $1000 \mathrm{ml}$ for the double strength medium. The $\mathrm{pH}$ of the medium should be adjusted to 6.5 with aqueous sodium hydroxide.

* Variable components.

to several other metabolites if tested in their medium which was already supplemented with alkali treated peptone. We repeated the experiment of Sarett and Cheldelin with asparagine, tryptophane, inositol and choline with the same basal medium except for omission of the alkali treated (or photolyzed) peptone supplement, and with the addition of high levels of thiamine. Whole yeast extract and norit treated yeast extract complements served for comparison. As seen on Table 3, there was no growth of $L$. fermenti with thiamine on the peptene-free basal medium lacking tryptophane. On the other hand, the addition of DL-tryptophane to the medium gave full growth which cannot be further increased with norit treated yeast extract. Repetition of the experiment with $0.1 \%$ Tween 80 included in the medium does not alter the results. Photolyzed peptone used as supplement at similar levels gave results in agreement with those obtained with yeast extract.

Thus tryptophane must be considered as an essential nutrient for $L$. fermenti, and the addition of different thiamine-free natural extracts as supplements to the basal medium are mainly justified as sources for tryptophane. Since alkali treatment of natural extracts not only destroys thiamine, but in the mean time decreases the original tryptophane content (cf. Ref. ${ }^{19}$ ), the incorporation of pure tryptophane in the basal media for $L$. fermenti is preferable. 
Table 3. Tryptophane as an essential nutrient for Lactobacillus fermenti 36. Turbidimetric readings of growth expressed in per cent transmission ( $\% \mathrm{~T})$. Blanks were adjusted $100 \% \mathrm{~T}$.

Basal medium according to Sarett and Cheldelin ${ }^{1}$ with omission of the alkali-treated peptone component was used with medium size inoculum. Each value represents the mean of triplicate readings.

\begin{tabular}{|l|c|c|c|}
\hline $\begin{array}{l}\text { Test } \\
\text { solutions } \\
\text { added }\end{array}$ & $\begin{array}{c}\text { Wedia } \\
\text { trypto- } \\
\text { phane }\end{array}$ & $\begin{array}{c}\text { With 4 mg } \\
\text { DL-trypto- } \\
\text { phane per 10 ml }\end{array}$ & $\begin{array}{c}\text { With tryptophane } \\
\text { plus 10\% Norit } \\
\text { treated Yeast } \\
\text { Extract }\end{array}$ \\
\hline \hline $\begin{array}{l}\text { Thiamine } \\
0.25 \mu \mathrm{g} \text { per 10 ml }\end{array}$ & 99.5 & 40.5 & 49 \\
\hline $\begin{array}{l}\text { Bacto-Yeast Extract } \\
\text { 4 mg per ml }\end{array}$ & 49 & 39 & 47 \\
\hline \hline $\begin{array}{l}\text { Thiamine } \\
0.25 \mu \mathrm{g} \text { per 10 ml }\end{array}$ & as above but with $0.2 \%$ Tween 80 in each tube \\
\hline $\begin{array}{l}\text { Bacto-Yeast Extract } \\
4 \mathrm{mg} \text { per 10 ml }\end{array}$ & 48 & 42.5 & 45 \\
\hline
\end{tabular}

Experiments performed with faint inoculum

Inoculum size was found by several workers ${ }^{3,5}$ to be one of the crucial points in the thiamine assay with $L$. fermenti. We found it desirable to adjust the density of the inoculum turbidimetrically in each run in order to ensure standard conditions, instead of the simple dilution of the washed inoculum 10 to 25 times. In contrast to the recommended "medium strength inoculum" $(40 \% \mathrm{~T})$ a rather weak one was used in most of the present nutritional tests. Thus there were better possibilities for the detection of small changes in the growth of this organism due to stimulatory or inhibitory action of different supplements, which usually affect the initial growth.

Effect of yeast extract. When initial growth with thiamine standards was measured after $12 \mathrm{~h}$ of incubation on different supplemented media, a marked early growth stimulation was found with whole yeast extract as seen in Fig. 1. Since the stimulation was clearly correlated to different thiamine concentrations, it cannot be accounted for by the presence of excess thiamine in the yeast extract. Norit treatment removes the stimulating factor from the yeast extract. This points to the possibility that the factor in question might be one of the B-vitamin type of growth stimulants. (It will be shown in a later chapter that the stimulatory effect can probably be due to Lactobacillus Bulgaricus Factor.)

Similar early growth stimulation would be disturbing in the case of routine assay procedure if complex natural extracts must be tested. However, the effect was eliminated by using a medium strength inoculum. 


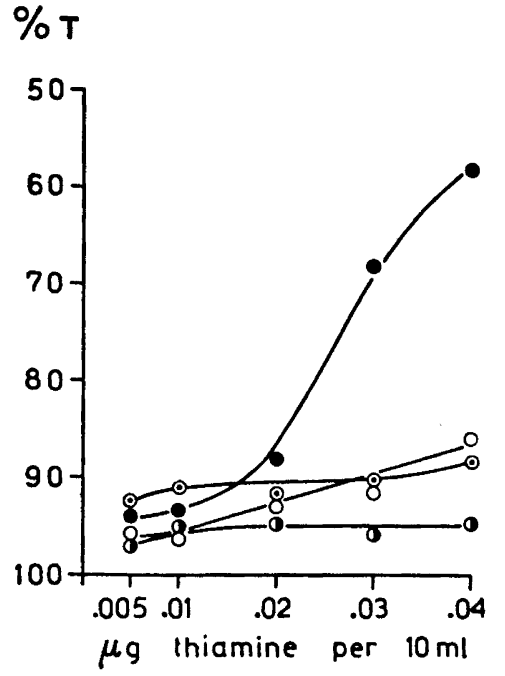

Fig. 1. Initial growth of $L$. fermenti 36 with thiamine standards using weak inoculum on different media: O Bacto Thiamine Assay Medium fortified with $0.1 \%$ Tween 80; $\odot$ the same with $10 \%$ Norit treated yeast extract added; (1) reduced low amino acid medium plus $10 \%$ Norit treated yeast extract; 0 reduced low amino acid medium with $2 \mathrm{mg}$ per tube BactoYeast-Extract added.

The effect of depleted yeast autolysates on the growth was considered for investigation because similar preparations were often recommended for use as supplement in assay media serving for sources of minute amounts of unidentified growth factors as well as of amino acids. Actually, thiamine-free yeast supplement was used in early work as a source of folic acid in the thiamine test ${ }^{1,12}$, and sulphite treated yeast extract was found by Bacharach and Cuthbertson ${ }^{4}$ to improve the visibility and diminish the "drift" when added to the Sarett and Cheldelin medium in the cup plate test. This contradicted the findings of Sarett and Cheldelin, who mention the trial with sulphite and Fuller's earth-treated yeast and liver extracts as a test for the lack of improvement in the assay of which was considered as proof for the completeness of their medium. Charcoal (Norit A) adsorption of yeast autolyzate solution originally introduced by Strong et al. ${ }^{16}$ was designed as a valuable supplement in the pantothenate test with Lactobacillus casei, a rather exacting organism. This preparation was tested by us with success in several microbiological assay media and is believed to be preferable to sulphite or alkali treated yeast extracts because these rather harsh treatments necessarily destroy many growth factors besides thiamine, leaving behind several split products and artefacts.

Contrary to the expectations the experiment with incorporation of Norittreated yeast extract in the basal media does not result in any improvement of the assay, but in marked inhibition of the growth of $L$. fermenti on both low and high amino acid unreduced media as seen in Fig. 2. Such inhibitory effect of a natural extract is evidently disturbing from the analytical viewpoint. Nevertheless, the inhibitory effect of the unknown substance(s) in the Norittreated yeast extract can be compensated by using reducing agents in the media, as seen in Fig. 3.

The effect of reducing agents, especially that of cysteine ${ }^{10}$ and of ascorbic acid 7,10 , on $L$. fermenti was studied thoroughly in the last years mainly be- 


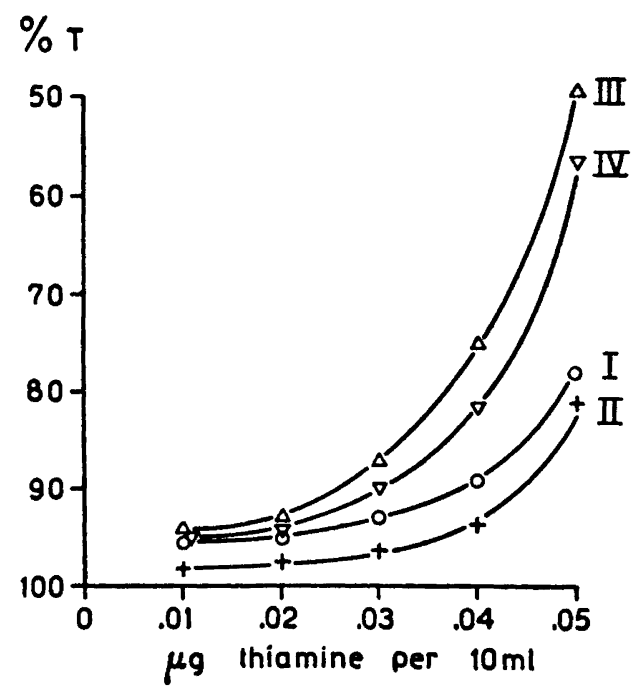

Fig. 2. Retardation of growth response of $L$. fermenti 36 toward thiamine by charcoal treated yeast extract with a weak inoculum. Curves I and II were obtained with low amino acid media, while III and IV with high amino acid media. Media in runs II and IV were supplemented with $10 \%$ Norit A treated Bacto-Yeast-Extract. Each value show the mean of readings in duplicates after $17 \mathrm{~h}$ incubation.

cause of expected improvement in the response of this organism toward thiamine. Reducing agents were found to enhance the growth especially in case of light inoculum and by using fructose or maltose instead of glucose. Ascorbic acid was claimed to act not only as a reducing agent, but also as a growth factor able to replace thiamine ${ }^{13}$. These findings were based again on the Sarett and Cheldelin basal medium with photolyzed peptone in it.

Testing the different semisynthetic media we found some stimulations with reducing agents in case of faint inoculum (cf. Figs. 3 and 4 ) which were equali-

Fig. 3. Compensation of the inhibitory effect of charcoal absorbed yeast extract supplement on the growth of $L$. fermenti 36 in the thiamine tube test by reducing agents. Weak inoculum was used and readings of duplicates were made after 16 $h$ of incubation on low amino acid media with: $\odot$ no supplement; $10 \%$ Norit A treated Bacto-Yeast Extract supplement; (1) the same as the latter supplemented with additional cysteine $(1 \mathrm{~g} / \mathrm{l})$ and ascorbic acid $(400 \mathrm{mg} / \mathrm{l})$.

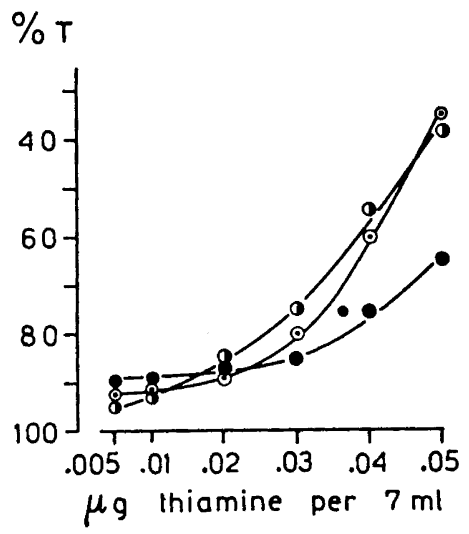




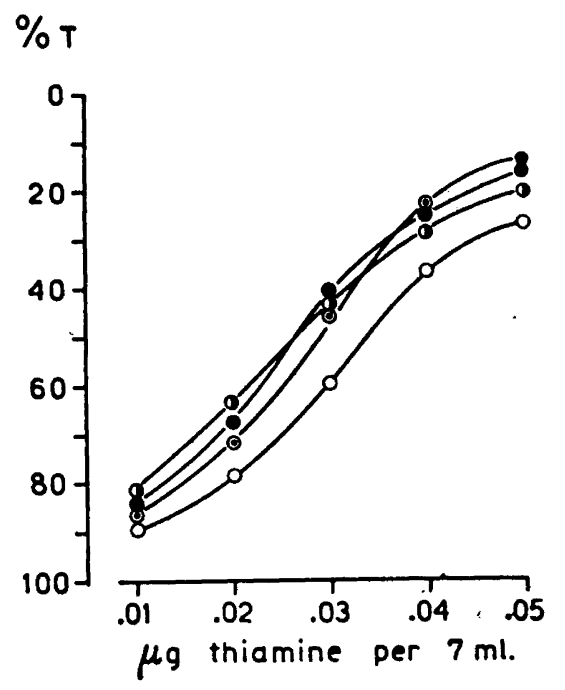

Fig. 4. Growth stimulation of $L$. fermenti 36 with pantethine. Experimental conditions similar to those for Fig. 3. Media: $O$ high amino acid medium; (1) the same with $25 \mu \mathrm{g}$ pantethine per liter final volume; $\odot$ reduced high amino acid medium; - the latter with pantethine as above.

zed by choosing suitable medium size inoculum (cf. Fig. 5). It was not possible to find any significant difference between the action of cysteine or ascorbic acid supposing that cystine was present in the semisynthetic media when testing ascorbic acid. Neither could we find a concentration-dependent thiamine-replacing effect of ascorbic acid on these media.

As seen in Fig. 3 the effect of reducing agents is rather marked when overcomming the inhibitory action of charcoal treated yeast extract. The beneficial effect of reducing agents is shown also in Fig. 4, where comparison of curves $\odot$ and $\odot$ gives a typical picture of growth stimulation by either cysteine or ascorbic acid or the combination, when $L$. fermenti was tested with thiamine on high amino acid medium, and the inoculum was weak.

Growth stimulation with Lactobacillus Bulgaricus Factor (LBF). As was already pointed out in connection with the interpretation of the experiment presented in Fig. 1, whole yeast extract contains some stimulatory factor(s) effective for early growth of weak inoculum. Because this type of growth stimulant might under certain conditions affect the thiamine assay, extended work seemed to be desirable at that point. Since the earlier nutritional studies with $L$. fermenti ${ }^{1},{ }^{13},{ }^{10}$, several new growth factors and stimulatory substances - mainly B vitamins - were detected partly with the aid of exacting Lactobacilli ${ }^{20}$. For this reason Vitamin $B_{12}$, Citrovorum Factor (Folinic acid), Thioctic acid (Protogen) and Lactobacillus Bulgaricus Factor (Pantethine) were tested by us. None of these factors showed additional growth stimulation with weak inocula on the media used in our work, except pantethine which is known to be a rather typical early growth stimulant for many lactic organisms, and with wide-spread occurrence.

As seen in Fig. 4 pantethine was found to be stimulatory for $L$. fermenti in a small but significant degree on a high amino acid medium with cystine. However, this stimulation was eliminated by incorporation of reducing agents in 
Fig. 5. Assay of thiamine standards with $L$. fermenti 36 on different media. Inoculum: 3 drops of $40 \% \mathrm{~T}$ suspension per $10 \mathrm{ml}$ media. Each value represents the mean of duplicate readings after $17 \mathrm{~h}$ of growth. Media 1-4, reduced high amino acid media with alkali treated peptone (20 g per l) in I ( $\triangle \triangle$ ); Norit A treated Bacto- Yeast Extract (10\%) in 2 $(\nabla--\nabla)$; Pantethine (10 $\mu \mathrm{g}$ per 1$)$ in 3 (0-- ; ); without supplement in 4 $(\mathrm{O}-\mathrm{O})$. For comparison in $5(+-\cdots++)$ a reduced low amino acid medium was used.

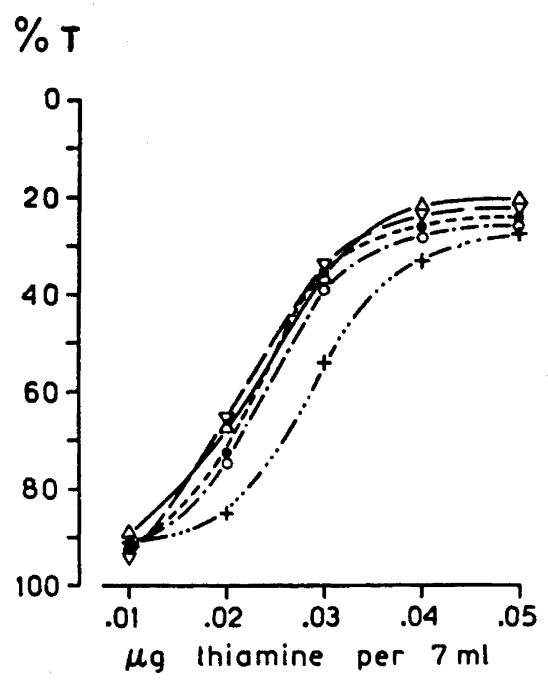

the medium. No such enhancing effect of pantethine was found to take place with more dense inocula. However, the incorporation of pantethine in a basal medium for analytical work with $L$. fermenti is still recommended.

The above results seem to be at first sight in contradiction with those of Craig and Snell ${ }^{21}$, who found an activity coefficient of less than 0.05 with pantethine for $L$. fermenti compared with pantothenic acid as unit. Still this result cannot be compared directly with ours, since the medium used by these authors was different from that of ours (e.g. calcium-D-pantothenate was not incorporated in the Craig and Snell medium) and the conditions of the inocula were not specified.

Thiamine assay with medium size of inoculum

Considering the important role of the inoculum for the assay of thiamine with $L$. fermenti, a final comparison was made with inocula of $40 \%$ transmission (2 drops per $7 \mathrm{ml}$ ) in different media. As seen in Fig. 5 the addition of different supplements does not influence the assay when the amount of acidhydrolyzed vitamin-free casein is increased to a level common for vitamin assay media ( $12 \mathrm{~g}$ instead of $5 \mathrm{~g}$ per liter final volume). The low amount of casein hydrolysate recommended by Sarett and Cheldelin ${ }^{1}$ was evidently calculated to be compensated by the incorporation of the peptone preparation (another source of amino acids). While the right size of the inoculum seems to be a limiting factor for reliable thiamine assay method for this organism on a semisynthetic medium, the composition of the medium is of great importance too. As a conclusion of the results presented herewith, a medium described in Table 2 is recommended for use. The addition of further supplements, though not essential, could be allowed except that the osmotic pressure of the final medium should not be too high to disturb the growth of $L$. fermenti. 
Table 4. The effect of the pyrimidine and thiazole moieties of thiamine on the growth of $L$. fermenti 36 in semisynthetic media. Turbidimetric readings, $24 \mathrm{~h}$ of growth.

\begin{tabular}{|c|c|c|c|c|c|}
\hline \multirow[t]{2}{*}{ Media } & \multirow{2}{*}{$\begin{array}{l}\text { Low amino acid } \\
\text { medium with } \\
10 \% \text { Norit A } \\
\text { treated Bacto- } \\
\text { Yeast Extract }\end{array}$} & \multicolumn{4}{|c|}{$\begin{array}{c}\text { High amino acid media with } \\
0.1 \% \text { Tween } 80\end{array}$} \\
\hline & & $\begin{array}{c}\text { Tween } \\
80 \\
\text { omitted }\end{array}$ & - & $\begin{array}{c}\text { with } 10 \mathrm{mg} \\
\text { per liter } \\
\text { Leuco- } \\
\text { vorine }\end{array}$ & $\begin{array}{l}\text { with } 10 \% \\
\text { Norit A } \\
\text { treated } \\
\text { Bacto-Yeast }\end{array}$ \\
\hline $\begin{array}{l}\text { 2-Methyl-4-amino-methyl } \\
\text { pyrimidine-hydrochloride } \\
0.03 \mu \mathrm{g} \text { per } 7 \mathrm{ml}\end{array}$ & $\begin{array}{l}95 \\
43\end{array}$ & $\begin{array}{l}98.5 \\
98\end{array}$ & $\begin{array}{l}98 \\
31.5\end{array}$ & $\begin{array}{l}97 \\
94.5\end{array}$ & $\begin{array}{l}96 \\
98\end{array}$ \\
\hline $\begin{array}{l}\text { 4-Methyl-5(-oxyethyl)- } \\
\text { thiazole } \\
0.03 \mu \mathrm{g} \text { per } 7 \mathrm{ml}\end{array}$ & $\begin{array}{l}30 \\
94.5\end{array}$ & 99 & $\begin{array}{l}17.5 \\
93\end{array}$ & 93.5 & $\begin{array}{l}94.5 \\
94.5\end{array}$ \\
\hline
\end{tabular}

The growth of $L$. fermenti with the pyrimidine and thiazole moieties of the thiamine molecule in absence of thiamine is one of the nutritional aspects which also needed to be controlled in case of introduction of a new medium. These moieties often interfere with microbiological procedures since different organisms can use one or both of these substances as precursors for the synthesis of thiamine. $L$. fermenti was reported to be unable to utilize either the pyrimidine or the thiazole parts under the first 20 to $24 \mathrm{~h}$ of growth, but seems to be stimulated by them during prolonged incubation ${ }^{1,5}$. Owing to this fact, the measurement of the growth of $L$. fermenti must take place before $18 \mathrm{~h}$ of incubation, which necessitates the use of turbidimetric readings instead of acidimetry. We tested both halves of thiamine on different media and found no growth after $20 \mathrm{~h}$ of incubation, while readings at $24 \mathrm{~h}$ showed occasional irregular randomly distributed growth, which points to the possibility of an adaptational mechanism (see Table 4) instead of direct utilization as is commonly believed.

Addendum: After the preparation of the present paper, the work of MaciasR ${ }^{22}$ was considered, in which a new thiamine test method with $L$. fermenti was presented. This method is based on a medium containing 5 to 400 times higher concentrations of the different $B$ vitamin components than the conventional levels. MaciasR's investigations were focused mainly on the carbohydrate requirements of $L$. fermenti, in variance with our present work. However, the experiments of MaciasR were conducted on the complete Sarett and Cheldelin medium, the disadvantages of which were discussed by us. Efforts to reproduce the work of MaciasR gave no satisfactory results in our laboratory.

The valuable assistance of Miss E. Börje and the financial support of the Swedish Natural Science Research Council is acknowledged.

\section{REFERENCES}

1. Sarett, H. P. and Cheldelin, V. H. J. Biol. Chem. 155 (1944) 153.

2. Barton-Wright, E. C. Analyst 70 (1945) 283.

3. Cheldelin, V. H., Bennett, M. J. and Kornberg, H. A. J. Biol. Chem. 166 (1946) 779. 
4. Bacharach, A. L. and Cuthbertson, W. F. J. Analyst 73 (1948) 334.

5. Fitzgerald, E. E. and Hughes, E. B. Analyst 74 (1949) 340.

6. Camien, N.N., Dunn, N.S. and Salle, A. J. J. Biol. Chem. 168 (1947) 33.

7. Fang, S. C. and Butts, J. S. Proc. Soc. Exptl. Biol. Med. 78 (1951) 463.

8. Dellweg, H. and Bernhauer, K. Biochem. Z. 328 (1956) 264.

9. Analytical Methods Committeo, Analyst 79 (1954) 118.

10. Snell, N. and Lewis, J. C. J. Bacteriol. 65 (1953) 671.

11. Bánhidi, Z. G., Sjöström, A. G. M., Grömmer, S. and Lundin, H. Arkiv Kemi 5 (1953) 393.

12. Barton-Wright, E. C. The Microbiological Assay of the Vitamin B-Complex and Amino Acids, London 1952.

13. Fang, S. C. and Butts, J. S. Proc. Soc. Exptl. Biol. Med. 82 (1953) 617.

14. Pennington, D., Snell, E. E. and Williams, R. J. J. Biol. Chem. 135 (1940) 213.

15. Snell, E. E. and Strong, F. M. Ind. Eng. Chem. Anal. Ed. 14 (1939) 346.

16. Strong, F. M., Geeney, R. E. and Earle, A. Ind. Eng. Chem. Anal. Ed. 13 (1941) 566.

17. Schultz, A. S., Atkin, L. and Frey, C. N. Ind. Eng. Chem. Anal. Ed. 14 (1942) 35.

18. Prescott, J. M., Schweigert, B. S., Lymen, C. M. and Kuiken, K. A. J. Biol. Chem. 178 (1949) 727.

19. Wooley, J. G. and Sebrell, W. H. J. Biol. Chem. 157 (1945) 141.

20. Kitay, E. and Snell, E. E. J. Bact. 60 (1950) 49.

21. Craig, J. A. and Snell, E. E. J. Bact. 61 (1951) 283.

22. MaciasR, F.M. Appl. Microbiol. 5 (1957) 249.

Received December 20, 1957. 